\title{
Was tun, wenn Metformin nicht ausreicht?
}

Zur unverzichtbaren Basistherapie bei allen Typ-2-Diabetikern gehört Metformin. Wenn mit dieser Substanz die geforderten Zielwerte nicht erreicht werden können, muss die Therapie erweitert werden. Nach den offiziellen Leitlinien der Deutschen Diabetes Gesellschaft sind verschiedene Optionen möglich.

- Im Rahmen einer Metaanalyse, in die die Ergebnisse von 27 randomisierten kontrollierten Studien eingingen, wurde die Wirksamkeit und Sicherheit der verschiedenen Therapieprinzipien, die dann zum Einsatz kamen, wenn die Therapie mit Metformin nicht ausreichte, miteinander verglichen, nämlich Glitazone, Sulfonylharnstoffe, Glinide, Alpha-Glukosidasehemmer, Gliptine und GLP-1-Analoga.

Bezüglich des $\mathrm{HbA}_{1 \mathrm{c}}$-Wertes zeigte sich kein relevanter Unterschied zwischen den einzelnen Substanzgruppen, d.h. es wurde mit allen Substanzen eine vergleichbare Abnahme des $\mathrm{HbA}_{1 c^{-}}$ Wertes von $0,64-0,87 \%$ erreicht. Doch bezüglich der Gewichtszunahme gab es durchaus Unterschiede. Während unter Glitazonen, Sulfonylharn-

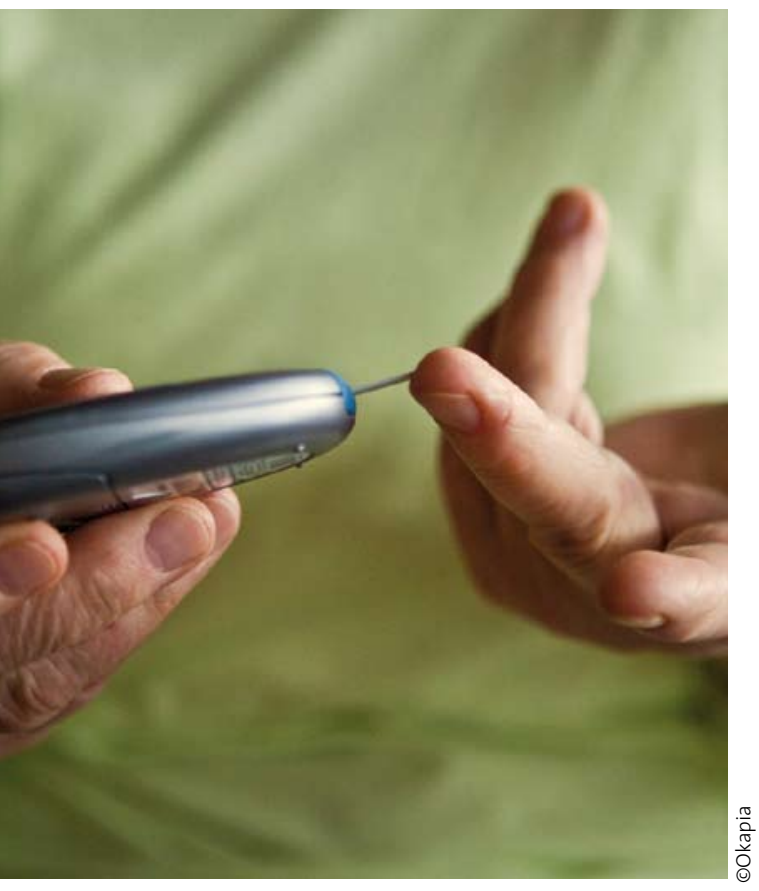

Ältere Diabetiker brauchen eine Therapie mit niedrigem Hypoglykämierisiko. stoffen und Gliniden eine Gewichtszunahme von 1,77-2,08 $\mathrm{kg}$ beobachtet wurde, fand man unter den anderen Therapiestrategien eine Gewichtsabnahme oder zumindest ein gleichbleibendes Gewicht. Bezüglich der Therapiesicherheit traten unter Sulfonylharnstoffen und Gliniden signifikant häufiger Hypoglykämien auf.

\section{Kommentar}

Oh, wie war es ehedem, doch nur mit Sulfonylharnstoffen noch so bequem! Die Einführung neuer innovativer antidiabetischer Substanzen hat die Therapie des Typ-2-Diabetes sehr viel komplexer gemacht. So gilt es, bei jedem einzelnen Patienten die Vor- und Nachteile einer bestimmten Substanzklasse abzuwägen. Dabei müssen auch die Nebenwirkungen und die Kontraindikationen Berücksichtigung finden. So sind Glitazone bei herzinsuffizienten Patienten und Sulfonylharnstoffe bei Patienten mit fortgeschrittener chronischer Niereninsuffizienz streng kontraindiziert. Nach neueren Studien dürfte das Hypoglykämierisiko insbesondere bei Patienten mit länger bestehendem Diabetes ein entscheidender prognostischer Faktor sein, da im Rahmen von Hypoglykämien vital bedrohliche kardiovaskuläre und rhythmogene Ereignisse auftreten können. Daraus ergibt sich die Notwendigkeit, gerade bei älteren Diabetikern vorzugsweise Substanzen einzusetzen, die das Hypoglykämierisiko nicht erhöhen. Dazu gehören vor allem die Gliptine und die GLP-1-Analoga.

Dr. med. Peter Stiefelhagen

\section{- O. J. Phung et al.}

Effect of noninsulin antidiabetic drugs added to metformin therapy on glycemic control, weight gain and hypglycemia in type 2 diabetes. JAMA 303 (2010) 1410-1418 